\title{
Physician burnout may contribute to racial bias
}

\author{
Cite as: CMAJ 2019 August 26;191:E951. doi: 10.1503/cmaj.109-5804
}

Posted on cmajnews.com on August 07, 2019.

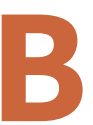

urned out doctors may be more prone to racial bias, according to a Mayo Clinic study published in JAMA Network Open.

The study measured racial bias and self-reported symptoms of burnout among 3380 second-year resident doctors in the United States who self-identified as nonblack. Researchers asked the residents to rate their feelings towards black or white people on a scale from cold to warm. They also tested for unconscious prejudice by measuring how quickly the residents sorted pictures of people of different races by positive or negative traits. Residents who reported symptoms of burnout scored higher on both measures of racial bias. Following up a year later with 2733 participants, a relationship was found between worsening of depersonalization and increases in negative attitudes toward black people.

These findings suggest that high rates of burnout in the medical profession may be contributing to health disparities, the authors concluded. "The implications for the quality of care provided to black people and other disadvantaged groups could be substantial."

"Physicians with higher implicit bias toward black people demonstrate fewer patient-centred behaviors during clinical interactions with black patients," wrote the authors. "In turn, their black patients have greater distrust, have lower levels of adherence to treatment recommendations, and are less likely to follow up."

Although there is growing recognition of the threat burnout poses to the safety and quality of care, not much is known about how to reduce rates among physicians, they suggested.

Residents in the Mayo Clinic study generally became less prejudiced over time, and those who recovered from burnout experienced the greatest drop in racial bias. This suggests a "doseresponse" link between burnout and bias, according to the authors of an accompanying commentary. However, it's not clear from the study whether burnout contributes to racial bias or vice versa, they wrote. "This is why addressing both burnout and bias together will be key to breaking the cycle."

Lauren Vogel, CMAJ

High rates of burnout among doctors may be contributing to racial disparities in health care, researchers warn. 